\title{
Apsidal motion in the eclipsing binary AS Camelopardalis
}

\author{
M. Wolf ${ }^{1}$, L. Šarounová ${ }^{1}$ and R. Diethelm ${ }^{2}$ \\ 1 Astronomical Institute, Charles University Prague, CZ-150 00 Praha 5, Švédská 8, Czech Republic \\ Electronic mail: wolf @ earn.cvut.cz \\ 2 Astronomical Institute, University Basel, CH-4102 Binningen, Venusstrasse 7, Switzerland
}

Received July 27; accepted October 7, 1995

\begin{abstract}
We report several new reliable times of minimum of the eclipsing binary AS Cam. Using all precise timings found in the literature, the $\mathrm{O}-\mathrm{C}$ diagram is analyzed and improved values for the elements of the apsidal motion are computed. The discrepancy between observed rotational velocity of the line of apsides and the theoretically expected value still remains unresolved.
\end{abstract}

Key words: binaries: eclipsing — stars: individual: AS Cam

\section{Introduction}

The analysis of apsidal motion in eclipsing binaries is not known only as an important source of information for the study of stellar interiors, but moreover, in some cases it serves also as a suitable test for the general theory of relativity.

AS Camelopardalis $\left(\mathrm{HD} 35311=\mathrm{BD}+69^{\circ} 325=\mathrm{SAO}\right.$ $13507=$ BV $268=$ GSC 4347.418) is an 8th magnitude double-line spectroscopic and eclipsing binary consisting of two main sequence B stars (B8V and B9.5V) in an eccentric orbit $(e=0.14)$ with orbital period of 3.43 days. It is an important system for the study of relativistic apsidal motion. As was shown by Maloney et al. (1989), this binary exhibits a discrepancy between observed and predicted rate of the apsidal motion. The theoretically expected rotational velocity of the line of apsides should be $44^{\circ} / 100 \mathrm{yr}$, caused by tidal distortion and rotational flattening of the component stars as well as by relativistic contribution.

AS Cam was discovered as a variable star photographically by Strohmeier (1959), who obtained also the first photographic light curve and determined the period of the system (Strohmeier \& Knige 1960). The first threecolor photometric study of this eclipsing binary, as well as the spectroscopic analysis were presented by Hilditch (1972a, b). Follow up photometric studies were published by Padalia \& Srivastava (1975) and Khaliulin \& Kozyreva (1983). Lines et al. (1989) and Maloney et al. (1989) were the first to announce that this system exhibits apsidal motion much less than expected from the general

Send offprint requests to: M. Wolf theory of relativity and from classical effects. The rate of apsidal motion was observed to be approximately $15^{\circ} / 100$ yr, about one third of the expected value.

Recently, the apsidal motion of AS Cam was studied by Krzesiński et al. (1990), who explained the discrepant rate of apsidal motion by the smaller value of the eccentricity $e=0.10$. They also published a complete list of epochs of minimum light for AS Cam. Finally, Maloney et al. (1991) discussed the previous photometric and spectroscopic solutions and analyzed the available photometry and spectroscopy with the Wilson-Devinney method. They concluded that the value of the eccentricity should be in the range $0.14<e<0.16$. As in the similar case of DI Her, the cause for the observed large discrepancy between observations and theory remains an unresolved problem.

In a recent paper (Wolf \& Šarounová 1995), we presented the apsidal motion analysis of the eclipsing binary FT Ori, which is a less known case of rather slow apsidal motion with period of about 480 years.

\section{Observations of minimum light}

To enlarge the collection of minimum times, new observations of the system were carried out. Our new photoelectric and CCD observations were performed in two periods during the 1991 and 1995 seasons.

For the secondary minimum obtained at JD 2448481 we used a $35 \mathrm{~cm}$ Cassegrain reflector with a single-channel photoelectric STARLIGHT-1 photometer furnished with an unrefrigereted EMI 9924A tube and the blue standard Johnson $B$ filter at the R. Szafraniec Observatory in 
Metzerlen, Switzerland. For these observations we chose the same comparison star $\left(\mathrm{BD}+69^{\circ} 323\right)$ as Caton \& Burns (1993).

The later observations were performed at the Ondřejov Observatory using a $65 \mathrm{~cm}$ reflecting telescope with a SBIG ST-6 CCD-camera. The measurements were done using the standard Johnson $B$ filter with $30 \mathrm{~s}$ exposure time. In this case the stars GSC $4347.452(V=10.77$ mag) on the same frame as AS Cam served as a comparison star. The CCD data were reduced using software developed at the Ondřejov Observatory by P. Pravec and M. Velen, which was modified for variable stars differential photometry.

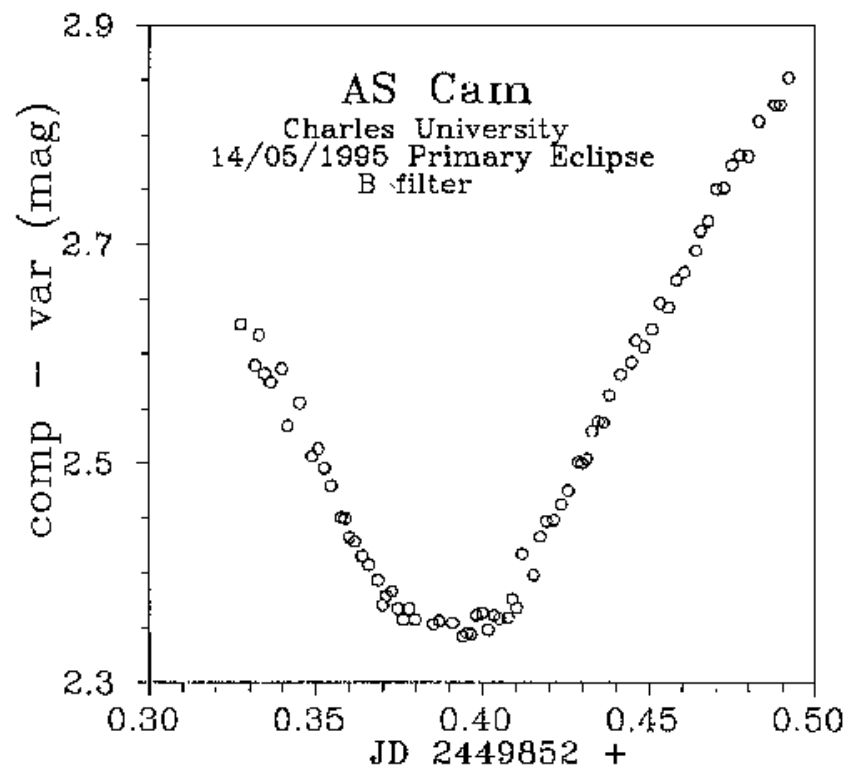

Fig. 1. A plot of the differential $B$-magnitudes obtained during primary eclipse of AS Cam on 14 May 1995

As an example of our CCD measurements, Fig. 1 shows the differential $B$-magnitudes during the primary minimum observed at JD 2449852.

No correction was allowed for differential extinction, due to the proximity of the comparison star to the variable and the resulting small differences in the air mass. This procedure is accurate enough for determination of a time of a minimum light. The new times of primary and secondary minimum and their error were determined using the Kwee-van Woerden (1956) method. They are presented in Table 1. In this table, $N$ stands for the number of observations symmetrically distributed with respect to the minimum and used in the calculations of minimum time. The epochs were calculated using the linear light elements given by Hilditch (1969):

Pri. Min. = HJD $2440204.5137+3.4309714 \cdot E$.
Table 1. New precise times of minimum of AS Cam

\begin{tabular}{lcccc}
\hline $\begin{array}{l}\text { JD Hel.- } \\
2400000\end{array}$ & $\begin{array}{c}\text { Error } \\
\left(10^{-4} \mathrm{~d}\right)\end{array}$ & Epoch & $N$ & Observatory \\
\hline 48481.5181 & 6 & 2412.5 & 45 & Metzerlen \\
49771.5636 & 8 & 2788.5 & 56 & Ondřejov \\
49852.3865 & 2 & 2812.0 & 61 & Ondřejov \\
49900.4177 & 5 & 2826.0 & 41 & Ondřejov \\
\hline
\end{tabular}

\section{Apsidal motion analysis}

The apsidal motion of AS Cam was studied by means of an O-C diagram analysis. All reliable times of minimum light were gathered from the literature by Krzesiński et al. (1990). In addition to these data, we considered the times of minimum obtained by Caton \& Burns (1993) as well as our own data.

The data reduction procedure was the following. All photoelectric times of minimum were used in our computation, with a weight of 10 , except the measurements of Couls (1989) $(E=2124)$, in which only the ascending branch of the light-curve is well covered, and the observations of Diethelm (1990) $(E=2241)$, which were measured during nonphotometric weather conditions. The weight of these both minima was reduced to 5 . The same weight was used for the normal photographic minima computed by Maloney et al. (1989). The other photographic times were given a weight of 1 or 0 , according to the list of minima in Krzesiński et al. (1990). The visual times of minimum were not used in our computation. A total of 63 times of minimum light were used in our analysis, with 24 secondary eclipses among them.

A numerical method for the apsidal motion analysis was described by Giménez \& García-Pelayo (1983). This method is a weighted least squares iterative procedure, including terms in the eccentricity up to the fifth order. Due to the small value of eccentricity we used only the terms up to the third order in our calculation. The formula for the prediction of the times of minimum $T_{j}$, used for the minimization by the least-squares method, is

$$
\begin{aligned}
T_{j} & =T_{0}+P_{\mathrm{s}} E+(j-1) \frac{P_{\mathrm{a}}}{2}+(2 j-3) \frac{e P_{\mathrm{a}}}{2 \pi}\left[2+\cot ^{2} i\right. \\
& \left.-\frac{e^{2} \cot ^{2} i}{4}\left\{3-\left(2+\csc ^{2} i\right) \csc ^{2} i\right\}\right] \cos \omega \\
& +\frac{e^{2} P_{\mathrm{a}}}{4 \pi}\left[\frac{3}{2}+\left(\csc ^{2} i+2\right) \cot ^{2} i\right] \sin 2 \omega \\
& -(2 j-3) \frac{e^{3} P_{\mathrm{a}}}{8 \pi}\left[\frac{4}{3}+\left(2+\csc ^{2} i\right) \cot ^{2} i \csc ^{2} i\right. \\
& \left.+3 \cot ^{2} i\right] \cos 3 \omega
\end{aligned}
$$

where $\omega=\dot{\omega} E+\omega_{0}$ and $j=1$ and 2 are taken for the primary and secondary minimum, respectively. In this equation $P_{\mathrm{a}}$ denotes the anomalistic period, $P_{\mathrm{s}}$ the sidereal 


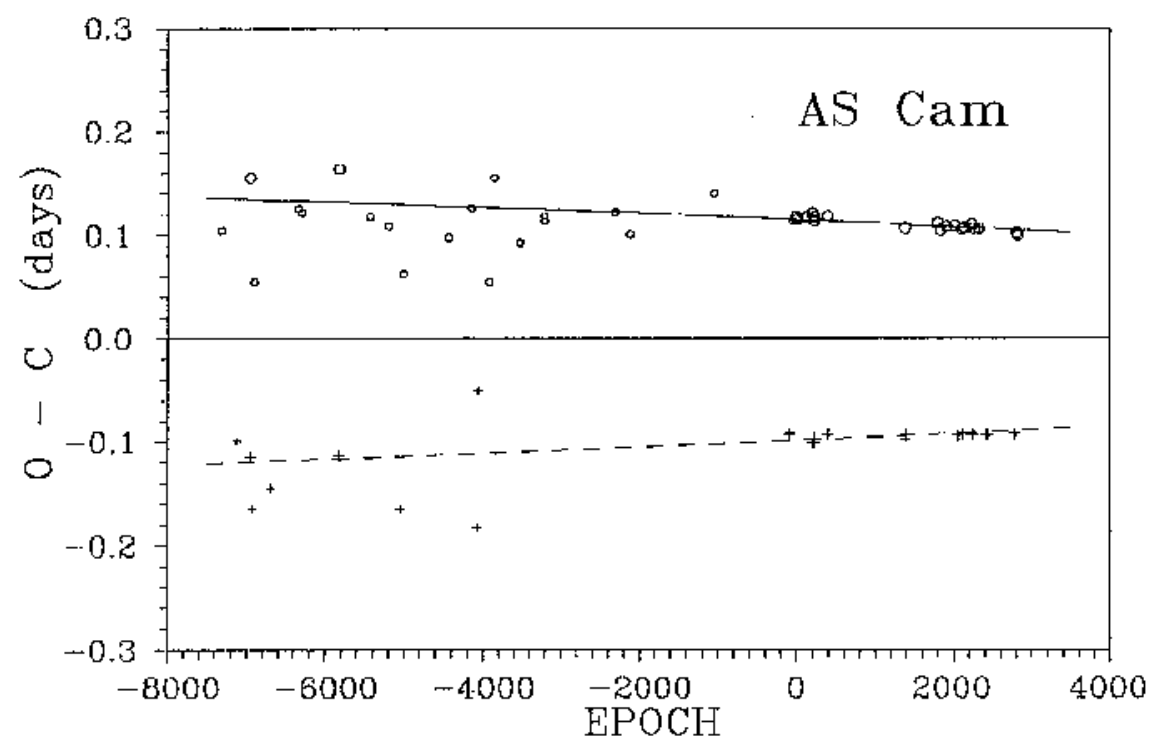

Fig. 2. Residuals for the times of minimum of AS Cam with respect to the linear part of the apsidal motion equation. The continuous and dashed curves represent predictions for primary and secondary eclipses, respectively. The individual primary and secondary minima are denoted by circles and crosses, respectively. Larger symbols correspond to the photographic and photoelectric measurements which were taken into calculations with higher weight

period, $e$ represents the eccentricity, $i$ the orbital inclination and $\dot{\omega}$ is the rate of periastron advance in degrees per cycle. The zero epoch is given by $T_{0}$ and the corresponding position of the periastron is represented by $\omega_{0}$. Equation (1) is also identical to the relation given by Todoran (1972). Fixing the value of eccentricity at $e=0.14$, there were four independent variables $\left(T_{0}, P_{\mathrm{s}}, \dot{\omega}, \omega_{0}\right)$ to be determined in this procedure. The relation between the two periods $P_{\mathrm{s}}$ and $P_{\mathrm{a}}$ is given by

$$
P_{\mathrm{s}}=P_{\mathrm{a}}\left(1-\dot{\omega} / 360^{\circ}\right)
$$

and the period of apsidal line rotation is then

$$
U=360^{\circ} P_{\mathrm{a}} / \dot{\omega}
$$

Comparing the different values of the orbital inclination found in the literature (Padalia \& Srivastava 1975; Khaliullin \& Kozyreva 1983; Maloney et al. 1991) we adopted the value of $i=88^{\circ}$. From this material mean apsidal motion elements given in Table 2 are computed; their standard errors are indicated.

The O-C residuals for all times of minimum with respect to the linear part of the apsidal motion equation are shown in Fig. 2. The original photographic times of minimum given by Hilditch (1969) before the zero epoch $T_{0}$ are also plotted. The non-linear predictions, corresponding to the fitted parameters, are plotted as continuous and dashed curves for primary and secondary eclipses, respectively.
Table 2. Apsidal motion elements of AS Cam

$$
\begin{aligned}
T_{0} & =2440204.3961 \pm 0.0004 \\
P_{\mathrm{s}} & =3.43097013 \pm 0.00000014 \mathrm{~d} \\
P_{\mathrm{a}} & =3.43098649 \pm 0.00000014 \mathrm{~d} \\
e & =0.14 \text { (fixed) } \\
\dot{\omega} & =(0.00172 \pm 0.00024)^{\circ} \mathrm{cycle}^{-1} \\
& =(0.183 \pm 0.026)^{\circ} \mathrm{yr}^{-1} \\
\omega_{0} & =226.0 \pm 0.1^{\circ} \\
U & =209000 P_{\mathrm{a}}=1970 \pm 280 \mathrm{yr}
\end{aligned}
$$

\section{Conclusions}

We computed the apsidal motion elements using the current data set. Our rate of apsidal motion is rather higher, but the results are in a good agreement with those previous presented by Maloney et al. (1989, 1991). The discrepancy between observed rotational velocity of the line of apsides and the theoretically expected value still remains unresolved.

Substracting the influence of apsidal motion, the $\mathrm{O}-\mathrm{C}_{2}$ diagram in Fig. 3 can be plotted. From this diagram no other phenomenon (i.e. presence of a third body in the system) can be simply derived. Nevertheless, the differences from the zero line are substancially larger than the standard errors of many observed times of minimum. Moreover, the $\mathrm{O}-\mathrm{C}_{2}$ residuals are almost positive for epochs 
$E<1000$ and negative for epochs $E>2000$ for both primary and secondary eclipses. For example, the lighttime effect with an amplitude about 0.005 day and a period over 10000 epochs (100 years) could be found in the residuals.

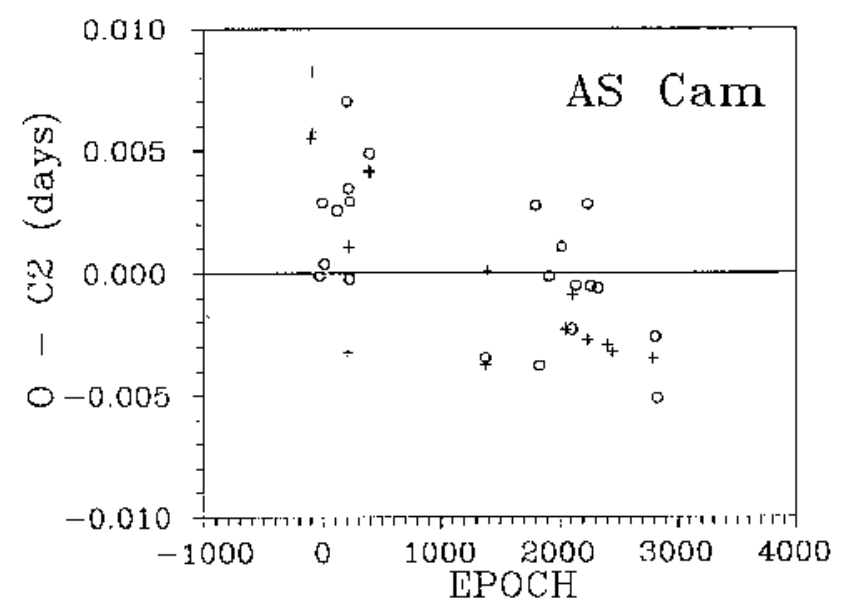

Fig. 3. $\mathrm{O}-\mathrm{C}_{2}$ residuals for the photoelectric times of minimum of AS Cam after subtraction the terms of the apsidal motion. The individual primary and secondary minima are denoted by circles and crosses, respectively

More high-accuracy timings of this eclipsing system are necessary in the future to enlarge the time span for a better analysis of the apsidal motion in this anomalous case and to resolve the question of additional influences on the O-C diagram.

For the current use we propose the following linear light elemets:

Pri. Min. = HJD $2440204.5134+3.4309654 \cdot E$

Sec. Min. = HJD $2440206.0167+3.4309706 \cdot E$
We plan to continue our photometric study of AS Cam and will attempt to obtain more precise photoelectric timings of primary and secondary eclipses in the next future.

Acknowledgements. This work has been supported in part by the Grant Agency of the Czech Republic, grant No. 205-951498 and by the ESO C\&EE Programme, grant No. A-02-069. This research has made use of the SIMBAD database, operated at CDS, Strasbourg, France.

\section{References}

Caton D.B., Burns W.C., 1993, Inf. Bull. Var. Stars No. 3900

Couls C.J., 1989, Inf. Bull. Var. Stars No. 3369

Diethelm R., 1990, BBSAG Bull. 93, 2

Giménez A., García-Pelayo J.M., 1983, Ap\&SS 92, 203

Hilditch R.W., 1969, Observatory 89, 143

Hilditch R.W., 1972a, PASP 84, 519

Hilditch R.W., 1972b, Mem. R. Astron. Soc. 76, 1

Khaliullin Kh.F., Kozyreva V.S., 1983, Ap\&SS 94, 115

Krzesiński J., Kuczawska E., Pajdosz G., 1990, Inf. Bull. Var. Stars No. 3495

Kwee K.K., Van Woerden H., 1956, Bull. Astron. Inst. Neth., 12,327

Lines H.C., Lines R.D., Glownia Z., Guinen E.F., 1989, PASP 101, 925

Maloney F.P., Guinan E.F., Boyd P.T., 1989, AJ 98, 1800

Maloney F.P., Guinan E.F., Mukherjee J., 1991, AJ 102, 256

Padalia T.D., Srivastava R.K., 1975, Ap\&SS 38, 87

Strohmeier W., 1959, Veröff. Rem. Sternw. Bamberg V, No. 3

Strohmeier W., Knige R, 1960, Veröff. Rem. Sternw. Bamberg VII, No. 72

Todoran I., 1972, Ap\&SS 15, 229

Wolf M., Šarounová L., 1995, A\&AS 114, 143 